\title{
Corrosion-resistance Microstructure Design using Mesoscale Modeling Environment for Additive Manufacturing for Co-Cr Alloys
}

\author{
Xiaoli Yan ${ }^{1}$, Arash Samei ${ }^{1}$, Brian Mercer $^{2}$, Pikee Priya $^{2}$, and Santanu Chaudhuri ${ }^{1 *}$ \\ 1. Department of Civil and Materials Engineering, University of Illinois at Chicago, Chicago, IL, USA. \\ 2. Department of Mechanical Science and Engineering, University of Illinois at Urbana-Champaign, \\ Urbana, IL, USA. \\ * Corresponding author: santc@uic.edu
}

This work-in-progress paper describes a combined framework for designing the selective laser melting (SLM) process for retaining microstructure and optimum corrosion performance. Since the SLM process and subsequent analysis of grain structure is time consuming, a closed-loop approach to microstructure control is currently not available. Addition of elements which gives a better control over corrosion process is key to many applications. In case of Co-Cr-Mo alloys, high corrosion resistance in cast component can be compromised due to SLM process. As cast ASTM F75 alloy usually exhibit a twophase microstructure consisting of $\sigma / \mathrm{M}_{23} \mathrm{C}_{6}$ and $\sigma / \mathrm{Co}-\alpha$-eutectic. Post-treatments such as heat treatment and aging are well-established for as-cast components to improve strength, wear and corrosion resistance. However, SLM manufactured parts needs to undergo hot isostatic press (HIP) post-treatment to consolidate the initial porous microstructure. Heat treatment and aging changes the carbide precipitates which are often main source of corrosion. Around the carbide grains, $\mathrm{Cr}$ is depleted due to galvanic corrosion. A strategy is thus needed which makes the precipitates in as printed parts and can be further improved by heat treatment. The modeling approach has two different components. First, we use our open source modeling software to obtain optimum grain size and orientation in the as-printed microstructure. Laser parameters can be adjusted for reducing precipitation in fine grained microstructures often produced during SLM. After HIP, the precipitates are often inside the grain, $\gamma$-fcc phase dominates the microstructure, and grain boundaries, compared to as-cast samples where the carbide phases are mostly found in the grain boundaries. This work is exploring two strategies for lowering the higher corrosion current in 3D printed parts. One strategy is to create conditions for minimizing the precipitation or dissolving precipitates. This can be done using our mesoscale SLM simulation and laser parameter optimization tool, MesMRISE. We will present results which explores strategies for increasing the desired Co $\gamma$-fcc phase in the as-printed samples. In addition, creating more equiaxed grains for better elongations can be beneficial. Modeling results for their relative grain orientations with respect to laser path and thermal gradients inside the melt pool will be discussed.The second strategy is adding elements which can improve corrosion resistance. In this case, the code we developed is called CRADLE for corrosion resistant microstructure design (Figure-1). One can compete with formation of the carbide phases which are responsible for corrosion and high strength. Among different carbide phases, some are more cathodic compare to other. We explore some preliminary hypothesis for corrosion control. It is possible to reform the distribution of carbides in Co-Cr-Mo-X-Y-Z type alloys which will lead to less corrosion. The most common carbide which forms during printing, HIPing, and aging is $\mathrm{M}_{23} \mathrm{C}_{6}$. Evidence for the presence of $\mathrm{MC}, \mathrm{M}_{3} \mathrm{C}_{7}, \mathrm{M}_{12} \mathrm{C}$, and $\mathrm{M}_{6} \mathrm{C}$ in the literature for Co-alloys is quite common. A first-principle based screening of elements and their carbides with will be analyzed to establish a balance between corrosion and strength.

In MesMRSE, a CFD model is implemented in OpenFoam which can be used to simulate melt pool flow and solidification during SLM additive manufacturing. The solver saves on computational cost by 
making modeling assumptions such as a continuum representation of powder regions, an optical penetration depth model for laser-powder interaction (as opposed to e.g. ray-tracing) while still capturing complex phenomenon like Marangoni flow driven by surface tension forces in the melt pool. Six different conditions for laser speed and power will be shown for melt pools in SLM-fabricated parts will be shown for differences in microstructure (Table-1). Such models can aid in optimizing laser scanning protocol and design microstructure within the limits of the SLM process. In CRADLE, an automated workflow is implemented to calculate formation enthalpy of precipitates, surface energy and work functions. The carbides with highest work function will deplete least amount of $\mathrm{Cr}$ from the intergranular regions through galvanic corrosion. Results will be demonstrated to compare different elements and their carbides for identifying most corrosion resistant intermetallic compounds. Since reactions needs to be favorable to redistribute the native carbides, different carbide formation reactions will be compared as alloy design strategy and analyzed against literature evidence from different Cobased superalloys.

\section{References:}

[1] Xiao, J., and S. Chaudhuri, Electrochimica Acta 56.16 (2011), p. 5630.

[2] Xiao, J., H. Kwak, and S. Chaudhuri, Proceedings of MS\&T 2011, Columbus, OH (2011), p. 1374.

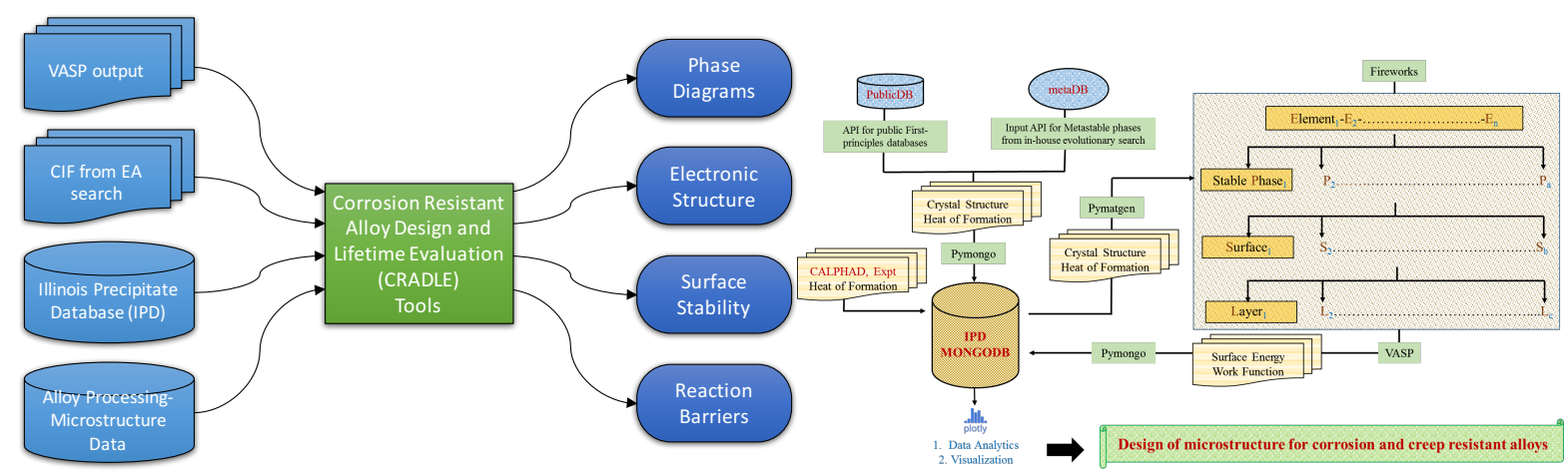

Figure 1. Corrosion resistance microstructure screening tool, CRADLE, and data flow is shown as schematic.

\begin{tabular}{|l|l|l|l|l|l|l}
\hline Case & Power $(\mathrm{W})$ & Speed $(\mathrm{mm} / \mathrm{s})$ & $\begin{array}{l}\text { Pool width } \\
\text { (microns) }\end{array}$ & Pool depth (microns) & Max pool velocity (cm/s) & M \\
\hline 1 & 300 & 900 & 273 & 55 & 238 & 7. \\
\hline 2 & 300 & 600 & 345 & 60 & 253 & 7. \\
\hline 3 & 280 & 1000 & 226 & 50 & 214 & 6. \\
\hline 4 & 250 & 800 & 250 & 50 & 210 & 5. \\
\hline 5 & 200 & 700 & 226 & 50 & 190 & 4. \\
\hline 6 & 200 & 1000 & 178 & 40 & & 175 \\
\hline
\end{tabular}

Table 1. Six combinations of laser power and speed are shown above for processing of Co-Cr-Mo alloys. The microstructure predictions and experimental measurements will be shown for comparison. 